\title{
LIPSCHITZIAN SOLUTIONS OF PERTURBED NONLINEAR PROGRAMMING PROBLEMS*
}

\author{
B. CORNET $\dagger$ AND J.-PH. VIAL $\dagger$
}

\begin{abstract}
We prove that if a second order sufficient condition and a constraint regularity assumption hold, then for sufficiently small perturbations of the constraints and the objective function, the set of local minimizers reduces to a singleton. Moreover, the minimizer and the associated multipliers are Lipschitzian functions of the parameter.
\end{abstract}

Key words. stability, nonlinear programming, weak convexity

AMS(MOS) subject classifications. 90C31, 90C30

1. Introduction. This paper deals with the stability of solutions and multipliers of nonlinear programming problems when the data are subjected to small perturbations. In order to formulate the problem, we introduce an open subset $U$ of $\mathbb{R}^{n}$, a metric space $P$, functions $f$ and $g$ from $U \times P$ to $\mathbb{R}$ and $\mathbb{R}^{m}$ and a nonempty closed subset $Q$ of $\mathbb{R}^{m}$. The problem of interest is then:

$$
\begin{array}{lll}
P(\alpha) \quad \text { minimize } & f(x, \alpha), \\
& \text { subject to } & g(x, \alpha) \in Q, \quad x \in U,
\end{array}
$$

where $x$ is the variable in which the minimization is done and $\alpha$ a perturbation parameter which belongs to $P$ and which remains fixed in the minimization problem.

We are interested in the behavior of local minimizers of $P(\alpha)$ when the parameter $\alpha$ varies. Our main result can be informally stated as follows. Under a set of assumptions dealing with (i) the smoothness of the functions $f$ and $g$, (ii) the regularity of the constraints at $(\bar{x}, \bar{\alpha})$, (iii) the weak convexity of the set $Q$ and (iv) a strong sufficient second-order condition at $(\bar{x}, \bar{\alpha})$, it is shown that, for small perturbations of the parameter $\bar{\alpha}$, the solution $\bar{x}$ of $P(\bar{\alpha})$ persists and is in Lipschitzian dependence with respect to the parameter. The importance of this Lipschitz property should be appreciated in the light of recent developments of calculus for Lipschitzian mappings (Clarke (1975), Rockafellar (1981)).

The above formulation of problem $P(\alpha)$ allows us to take into account the classical nonlinear programming problem with equality and/or inequality constraints, i.e., $Q=\{0\}^{m_{1}} \times\left(-\mathbb{R}_{+}^{m_{2}}\right)$ for nonnegative integers $m_{1}$ and $m_{2}$. The consideration of more general sets $Q$ in $P(\alpha)$ is motivated by the following property of weakly convex sets, a class of sets introduced by Vial (1983) (see also Cornet (1981)), which includes as special cases, convex subsets of $\mathbb{R}^{m}$ and twice continuously differentiable submanifolds of $\mathbb{R}^{m}$ with or without a boundary. Let $Q$ be a nonempty closed subset of $\mathbb{R}^{m}$ and let $\alpha$ be in $\mathbb{R}^{m}$; then the set of projections of $\alpha$ on $Q$, denoted $\pi(\alpha)=$ $\left\{x \in Q \mid\|x-\alpha\| \leqq\left\|x^{\prime}-\alpha\right\|\right.$, for all $x^{\prime}$ in $\left.Q\right\}$, clearly is the set of solutions of problem $P(\alpha)$ for well chosen mappings $f$ and $g$. An important property of weakly convex sets is that the mapping $\pi$ is single-valued and Lipschitzian on a neighborhood of $Q$. Our main theorem generalizes the known results for problems with equality and/or inequality constraints and also includes the above property of weakly convex sets.

\footnotetext{
* Received by the editors March 31, 1983, and in revised form May 1, 1985.

$\uparrow$ CORE, 1348 Louvain-La-Neuve, Belgium.
} 
We conclude this section by indicating the link between this paper and the rest of the literature. The standard problem with equality and/or inequality constraints has been studied by Fiacco and McCormick (1968), Robinson (1974), Fiacco (1976). The basic feature of these articles is that, under the strict complementarity slackness assumption, it is proved, using the standard implicit function theorem, that the stationary points (i.e., points that satisfy the first order necessary condition for optimality), and their associated multipliers are differentiable.

The strict complementarity slackness assumption has been removed, in the case of equality and/or inequality constraints by Robinson (1980), Kojima (1980), Jittorntrum (1984) and in the case where $Q$ is a closed convex subset of $\mathbb{R}^{m}$ by Cornet-Laroque (1986) (see also Cornet-Laroque (1980), Cornet (1981)) and J.-P. Aubin (1981) when the constraints are linear. In these cases, under a somewhat stronger second order sufficient condition, a similar result is shown to hold, namely that the stationary points and associated multipliers are (locally) Lipschitzian mappings of the perturbation. The main tool for these analyses are generalizations of the standard implicit function theorem; for example Robinson (1980) proves a general implicit theorem for "generalized equations" (i.e. of variational inequalities), Cornet-Laroque (1986) use a generalization of the implicit function theorem in the case of Lipschitzian mappings due to Clarke (1976) (see also Auslender (1983)) and J.-P. Aubin (1981) a generalization of it in the case of convex processes.

Our approach in the present paper is different from the previous ones. It is direct in the sense that no implicit function theorem or generalization of it is used. We are able to show the local persistence of a local minimizer of our problem and next the Lipschitzian dependence with respect to the parameter $\alpha$. The first step owes much to a result of Robinson (1982). Finally we shall mention the work of Levitin (1975) who made an analysis of the Lipschitz dependence of local minimizers, also by a direct approach. However, it contains an apparent error as is pointed out in the paper of Robinson (1982).

Our paper is organized as follows. In $\S 2$, we recall some definitions and state the main result of the paper. We also discuss two noteworthy applications: the first deals with the projection of points on a weakly convex set, and the second deals with the standard nonlinear programming problem. The proof of the main theorem is given in $\S 3$.

2. Statement of the main theorem and some consequences. Let us first introduce some notations and definitions. Let $x=\left(x_{i}\right), y=\left(y_{i}\right)$ be in $\mathbb{R}^{q}$; we denote $\langle x, y\rangle=$ $\sum_{i=1}^{q} x_{i} \cdot y_{i}$, the scalar product of $\mathbb{R}^{q}$, and $\|x\|=\langle x, x\rangle^{1 / 2}$ the Euclidean norm. Let $A$ be a nonempty subset of $\mathbb{R}^{q}$ and let $x$ be in $\mathbb{R}^{q}$; we denote $d_{A}(x)=\inf \{\|a-x\| \mid a \in A\}$, $B(A, \varepsilon)=\left\{x \in \mathbb{R}^{q} \mid d_{A}(x)<\varepsilon\right\}$ and $\bar{B}(A, \varepsilon)=\left\{x \in \mathbb{R}^{q} \mid d_{A}(x) \leqq \varepsilon\right\}$. Let $Q$ be a subset of $\mathbb{R}^{m}$ and let $x$ be in $\bar{Q}$; we recall the following definitions of Clarke (1975) of the tangent cone $T_{Q}(x)$ and the normal cone $N_{Q}(x)$ to $Q$ at $x$,

$$
\begin{aligned}
T_{Q}(x)=\left\{v \in \mathbb{R}^{m} \mid \text { for all sequences }\left\{\theta_{k}\right\} \subset(0, \infty) \text { and }\left\{x_{k}\right\} \subset \bar{Q} \text { such that } \theta_{k} \rightarrow 0,\right. & \\
& \left.x_{k} \rightarrow x, \text { there exists a sequence }\left\{v_{k}\right\} \rightarrow v \text { such that, for all } k, x_{k}+\theta_{k} v_{k} \in \bar{Q}\right\}, \\
N_{Q}(x)= & \left\{\eta \in \mathbb{R}^{m} \mid\langle\eta, v\rangle \leqq 0, \text { for all } v \in T_{Q}(x)\right\} .
\end{aligned}
$$

Definition 1 . A subset $Q$ of $\mathbb{R}^{m}$ is said to be weakly convex, with constant $\rho \geqq 0$, at an element $y^{0}$ in $\bar{Q}$, if there exists $\varepsilon>0$ such that, for all $y^{1}, y^{2}$ in $\bar{Q} \cap B\left(y^{0}, \varepsilon\right)$ and for all $\lambda^{2} \in N_{Q}\left(y^{2}\right) \cap \bar{B}(0,1)$, one has

$$
\left\langle\lambda^{2}, y^{2}-y^{1}\right\rangle \geqq-\frac{\rho}{2}\left\|y^{2}-y^{1}\right\|^{2} .
$$


It is possible to give the following geometric interpretation of weakly convex sets, of constant $\rho>0$. Let $Q$ be such a set and let $X=\bar{Q} \cap B\left(y^{0}, \varepsilon\right)$ with $y_{0} \in \bar{Q}$. Then for all $x \in X$ and $\eta \in N_{Q}(x) \cap \bar{B}(0,1)\left(=N_{X}(x) \cap \bar{B}(0,1)\right)$,

$$
X \cap B\left(x+\rho^{-1} \eta, \rho^{-1}\|\eta\|\right)=\varnothing .
$$

For $\eta \neq 0$, one could view $B\left(x+\rho^{-1} \eta, \rho^{-1}\|\eta\|\right)$ as a "supporting ball," very much in a sense analogous to a supporting hyperplane for a convex set. In this terminology, if a set is weakly convex at $y^{0}$, one can exhibit a "supporting ball" at each point of the boundary of the set in a neighborhood of $y^{0}$. Note that the radius of the "supporting ball" is fixed in the given neighborhood. Clearly, a convex subset $Q$ of $\mathbb{R}^{m}$ is weakly convex with respect to any constant $\rho \geqq 0$. We refer to Cornet (1981), Vial (1983) for other examples of weakly convex sets (such as $C^{2}$ submanifolds in $\mathbb{R}^{m}$ with or without a boundary) and/or for properties of weakly convex sets.

We posit the following assumptions, which describe the general framework of the paper.

Assumptions A.0

(i) $U$ is an open subset of $\mathbb{R}^{n} ; P$ is a metric space endowed with a distance $d$;

(ii) the functions $f(\cdot, \cdot)$ and $g_{i}(\cdot, \cdot), i=1, \cdots, m$, are locally Lipschitzian from $U \times P$ to $\mathbb{R}$

(iii) for all $\alpha \in P$, the functions $f(\cdot, \alpha)$ and $g_{i}(\cdot, \alpha), i=1, \cdots, m$, are twice continuously differentiable from $U$ to $\mathbb{R}$;

(iv) the mappings $\nabla f(\cdot, \cdot)$ and $\nabla g_{i}(\cdot, \cdot), i=1, \cdots, m$, of first partial derivatives with respect to the first argument, are locally Lipschitzian from $U \times P$ to $\mathbb{R}^{n}$;

(v) the mapping $D^{2} f(\cdot, \cdot)$ and $D^{2} g_{i}(\cdot, \cdot)$ of second order derivatives with respect to the first argument, are continuous;

(vi) $m=m_{1}+m_{2}$, where $m_{1}$ and $m_{2}$ are nonnegative integers; $C$ is a nonempty closed subset of $\mathbb{R}^{m_{2}}$ and $Q=\{0\}^{m_{1}} \times C$ (with the convention that $Q=\{0\}^{m}$ if $m_{2}=0$ and $Q=C$ if $\left.m_{1}=0\right)$.

We consider the following perturbed nonlinear programming problem:

$$
\begin{array}{cl}
\text { minimize } & g(x, \alpha), \\
P(\alpha) \quad \text { subject to } & g(x, \alpha) \in Q, \\
& x \in U,
\end{array}
$$

where $g(x, \alpha)$ is the vector in $\mathbb{R}^{m}$ with coordinates $g_{i}(x, \alpha), i=1, \ldots, m, x$ is the variable in which the minimization is done, and $\alpha \in P$ is a perturbation term which remains fixed in the minimization problem. Note that it is possible to rewrite the constraints as follows. For all $(x, \alpha)$ in $U \times P$, let $g_{E}(x, \alpha)$ (resp. $\left.g_{I}(x, \alpha)\right)$ be the vector in $\mathbb{R}^{m_{1}}$ (resp. $\mathbb{R}^{m_{2}}$ ) with coordinates $g_{i}(x, \alpha), i=1, \cdots, m_{1}\left(\right.$ resp. $\left.i=m_{1}+1, \cdots, m\right)$. Then $x$ satisfies the constraints of $P(\alpha)$ if and only if:

$$
g_{E}(x, \alpha)=0 \quad \text { and } \quad g_{I}(x, \alpha) \in C, \quad x \in U .
$$

With $P(\alpha)$, we associate the following "generalized equation":

$$
\begin{aligned}
& \nabla f(x, \alpha)+\sum_{i=1}^{m} \lambda_{i} \nabla g_{i}(x, \alpha)=0, \\
& g(x, \alpha) \in Q \text { and } \lambda=\left(\lambda_{i}\right) \in N_{Q}(g(x, \alpha)) .
\end{aligned}
$$

We shall be concerned with pairs $\left(x^{0}, \alpha^{0}\right) \in U \times P$ such that $x^{0}$ is a local minimizer of $P\left(\alpha^{0}\right)$. If we further assume that: 
Assumption A.1. The gradients $\nabla g_{i}\left(x^{0}, \alpha^{0}\right), i=1, \cdots, m$, are linearly independent, then we shall prove later (Lemma 3.1) that there exists $\lambda^{0} \in \mathbb{R}^{m}$ such that $\left(x^{0}, \alpha^{0}, \lambda^{0}\right)$ solves (2.1). In other words, (2.1) is the first order necessary condition associated with $P(\alpha)$. For such a triplet we posit the following two assumptions:

Assumption A.2. Q is weakly convex with constant $\rho \geqq 0$ at $g\left(x^{0}, \alpha^{0}\right)$. (Note that it would be equivalent to replace the above statement by " $C$ is weakly convex with constant $\rho \geqq 0$ at $g_{I}\left(x^{0}, \alpha^{0}\right)$.")

Assumption A.3. There exist real numbers $a \geqq 0$ and $c>0$ such that, for all $h \in \mathbb{R}^{n}$, one has

$$
\begin{aligned}
& \left\langle\left[D^{2} f\left(x^{0}, \alpha^{0}\right)+\sum_{i=1}^{m} \lambda_{i}^{0} D^{2} g_{i}\left(x^{0}, \alpha^{0}\right)\right] h, h\right\rangle \\
& \quad+a\left\langle\nabla f\left(x^{0}, \alpha^{0}\right), h\right\rangle^{2}+a \sum_{i=1}^{m_{1}}\left\langle\nabla g_{i}\left(x^{0}, \alpha^{0}\right), h\right\rangle^{2} \geqq c\|h\|^{2}
\end{aligned}
$$

Note that the index in the first sum runs from 1 to $m=m_{1}+m_{2}$ and from 1 to $m_{1}$ in the second. (A.3) clearly implies the more familiar assumption:

Assumption A.3'. For all $h \in \mathbb{R}^{n}, h \neq 0$, such that $\left\langle\nabla f\left(x^{0}, \alpha^{0}\right), h\right\rangle=0$ and $\left\langle\nabla g_{i}\left(x^{0}, \alpha^{0}\right), h\right\rangle=0, i=1, \cdots, m_{1}$, one has

$$
\left\langle\left[D^{2} f\left(x^{0}, \alpha^{0}\right)+\sum_{i=1}^{m} \lambda_{i}^{0} D^{2} g_{i}\left(x^{0}, \alpha^{0}\right)\right] h, h\right\rangle>0 .
$$

It is an immediate consequence of a lemma of Debreu (1952) that (A.3') implies (A.3). Thus the two assumptions are equivalent.

We can now state the main theorem.

Theorem 2.1. Assume (A.0) and let $\left(x^{0}, \alpha^{0}, \lambda^{0}\right) \in U \times P \times \mathbb{R}^{m}$ satisfy (A.1), (A.2), (A.3). Further, assume that the constants $\rho \geqq 0$ and $c>0$ satisfy

Assumption A.4. $c>\lambda_{I}^{0}\left\|D g_{I}\left(x^{0}, \alpha^{0}\right)\right\|^{2}$, where $\lambda_{I}^{0}=\left(\lambda_{m_{1}}^{0}+1, \cdots, \lambda_{m}^{0}\right)$.

Then, if $\left(x^{0}, \alpha^{0}, \lambda^{0}\right)$ satisfies condition (2.1), there exist neighborhoods $U^{\prime}$ of $x^{0}$ in $U, V^{\prime}$ of $\alpha^{0}$ in $P$ and mappings $x(\cdot): V^{\prime} \rightarrow U^{\prime}, \lambda(\cdot): V^{\prime} \rightarrow \mathbb{R}^{m}$ such that:

(i) $x(\cdot)$ and $\lambda(\cdot)$ are Lipschitzian;

(ii) $x\left(\alpha^{0}\right)=x^{0}$ and $\lambda\left(\alpha^{0}\right)=\lambda^{0}$;

(iii) for all $\alpha$ in $V^{\prime}, x(\alpha)$ is the unique minimizer of $P(\alpha)$ in $U^{\prime}$ and $\lambda(\alpha)$ is the unique Kuhn-Tucker multiplier associated with $x(\alpha)($ i.e., $(x(\alpha), \lambda(\alpha))$ satisfies condition (2.1)).

The proof of Theorem 2.1 is given in the next section.

Remark 1. Assumption (A.1) cannot be relaxed in the case of equality and/or inequality constraints (i.e., when $Q=\{0\}^{m_{1}} \times\left(-\mathbb{R}_{+}^{m_{2}}\right)$ ) by only assuming the Mangasarian-Fromovitz's constraint qualification (see Robinson (1980)).

Remark 2. If $C$ is convex, then (A.4) is trivially satisfied (since convex sets in $\mathbb{R}^{m_{2}}$ are weakly convex with constant $\rho=0$ ). It is worth pointing out that in the case of equality and/or inequality constraints, (A.3) is stronger than the classical sufficient second-order condition of Fiacco and McCormick (1968). However, Theorem 2.1 does not hold if one replaces (A.3) by the classical second order condition as it has been shown by Robinson (1980).

We conclude this section by discussing two noteworthy applications of Theorem 2.1. The first one deals with the projection mapping on a weakly convex subset of $\mathbb{R}^{n}$.

Let $Q$ be a nonempty closed subset of $\mathbb{R}^{n}$. For a fixed element $\alpha$ in $\mathbb{R}^{n}$, we consider the following minimization problem: 
$\begin{array}{lll}R(\alpha) & \text { minimize } & \frac{1}{2}\|x-\alpha\|^{2}, \\ & \text { subject to } & x \in Q,\end{array}$

and we denote by $\pi(\alpha)$ the set of its solutions. Any element of $\pi(\alpha)$ is called a projection of $\alpha$ on $Q$. The next proposition gives some properties of the (multi-valued) mapping $\alpha \rightarrow \pi(\alpha)$ when $Q$ is assumed to be weakly convex.

COROllary 2.2. Let $\left(x^{0}, \alpha^{0}\right) \in \mathbb{R}^{n} \times \mathbb{R}^{n}$ be such that $x^{0}$ is a local minimizer of $R\left(\alpha^{0}\right)$. Assume that $Q$ is weakly convex with constant $\rho \geqq 0$ at $x^{0}$ and that $1>\rho\left\|x^{0}-\alpha^{0}\right\|$. Then, there exist neighborhoods $U^{\prime}$ of $x^{0}, V^{\prime}$ of $\alpha^{0}$ and a Lipschitzian mapping $x(\cdot): V^{\prime} \rightarrow U^{\prime}$ such that $x\left(\alpha^{0}\right)=x^{0}$ and, for all $\alpha \in V^{\prime}, x(\alpha)$ is the unique minimizer of $R(\alpha)$ in $U^{\prime}$.

Proof. It is a trivial matter to check that (A.1) and (A.3) are satisfied with $c=1$ and that the Kuhn-Tucker multiplier $\lambda^{0}$ associated with $x^{0}$ satisfies $\lambda^{0}=-\left(x^{0}-\alpha^{0}\right)$. Thus (A.4) reduces to $1=c>\rho\left\|x^{0}-\alpha^{0}\right\|$. Hence the result.

Remark 3. When $m_{1}=0$, we give here an example showing that the inequality in (A.4) is the best possible. Let $Q=\left\{x \in \mathbb{R}^{m} \mid\|x\| \geqq 1\right\}$; clearly, $Q$ is weakly convex at every element $x$ in $Q$, with constant $\rho=1$. If $\alpha^{0} \neq 0$, let $\mu=\max \left\{1,\left\|\alpha^{0}\right\|^{-1}\right\}$, then $x^{0}=\mu \alpha^{0}$ is the unique minimizer of $R\left(\alpha^{0}\right)$. Since the hypotheses of Corollary 2.2 are satisfied at $x^{0}$, the conclusion of the corollary holds. However, if $\alpha^{0}=0$, any $x^{0}$ such that $\left\|x^{0}\right\|=1$ is a minimizer of $R\left(\alpha^{0}\right)$. Obviously, $1=\rho\left\|x^{0}-\alpha^{0}\right\|$; hence Assumption (A.4) is violated and one easily sees directly that the conclusion of Corollary 2.2 cannot hold.

The second application deals with standard nonlinear programming. Assume A.0 and assume furthermore that $Q=\{0\}^{m_{1}} \times\left(-\mathbb{R}_{+}^{m_{2}}\right)$ (i.e., in (vi) of (A.0), $\left.C=-\mathbb{R}_{+}^{m_{2}}\right)$. We consider the standard perturbed nonlinear programming problem:

$$
\begin{array}{ll}
\operatorname{minimize} & f(x, \alpha), \\
\text { subject to } & g_{i}(x, \alpha)=0, i=1, \cdots, m_{1}, \\
& g_{i}(x, \alpha) \leqq 0, i=m_{1}+1, \cdots, m, \\
& x \in U .
\end{array}
$$

With $S(\alpha)$, we associate the first order necessary conditions:

$$
\begin{aligned}
& \nabla f(x, \alpha)+\sum_{i=1}^{m_{1}+m_{2}} \lambda_{i} \nabla g_{i}(x, \alpha)=0, \\
& g_{i}(x, \alpha)=0, \quad i=1, \cdots, m_{1}, \\
& g_{i}(x, \alpha) \leqq 0, \quad \lambda_{i} \geqq 0, \quad \lambda_{i} g_{i}(x, \alpha)=0, \quad i=m_{1}+1, \cdots, m_{1}+m_{2} .
\end{aligned}
$$

In the case of standard nonlinear programming, Assumptions (A.2) and (A.4) are satisfied, since $Q=\{0\}^{m_{1}} \times\left(-\mathbb{R}_{+}\right)^{m_{2}}$ is convex. We give now a consequence of Theorem 2.1, where the Assumptions (A.1) and (A.3) are weakened. First, let us introduce the following notation: for $(x, \alpha) \in U \times P$, satisfying the constraints of $S(\alpha)$, we let $I(x, \alpha)=$ $\left\{i \in\left\{m_{1}+1, \cdots, m\right\} \mid g_{i}(x, \alpha)=0\right\}$ be the set of active inequality constraints.

Corollary 2.3. Assume (A.0) and let $\left(x^{0}, \alpha^{0}, \lambda^{0}\right) \in U \times P \times \mathbb{R}^{m}$ be such that:

(C.1) the vectors $\nabla g_{i}\left(x^{0}, \alpha^{0}\right), i \in\left\{1, \cdots, m_{1}\right\} \cup I\left(x^{0}, \alpha^{0}\right)$, are linearly independent;

(C.2) for all $h \in \mathbb{R}^{n}, h \neq 0$, satisfying $\left\langle\nabla f\left(x^{0}, \alpha^{0}\right), h\right\rangle=0$ and $\left\langle\nabla g_{i}\left(x^{0}, \alpha^{0}\right), h\right\rangle=0, i \in$ $\left\{1, \cdots, m_{1}\right\} \cup\left\{i \in I\left(x^{0}, \alpha^{0}\right) \mid \lambda_{i}^{0}>0\right\}$, then

$$
\left\langle\left[D^{2} f\left(x^{0}, \alpha^{0}\right)+\sum_{i=1}^{m} \lambda_{i}^{0} D^{2} g_{i}\left(x^{0}, \alpha^{0}\right)\right] h, h\right\rangle>0 \text {. }
$$


Then, if $\left(x^{0}, \alpha^{0}, \lambda^{0}\right)$ satisfies condition (2.2), there exist neighborhoods $U^{\prime}$ of $x^{0}$ in $U, V^{\prime}$ of $\alpha^{0}$ in $P$ and mappings $x(\cdot): V^{\prime} \rightarrow U^{\prime}, \lambda(\cdot): V^{\prime} \rightarrow \mathbb{R}^{m}$ such that:

(i) $x(\cdot)$ and $\lambda(\cdot)$ are Lipschitzian;

(ii) $x\left(\alpha^{0}\right)=x^{0}$ and $\lambda\left(\alpha^{0}\right)=\lambda^{0}$;

(iii) for all $\alpha \in V^{\prime}, x(\alpha)$ is the unique minimizer of $S(\alpha)$ in $U^{\prime}$ and $\lambda(\alpha)$ is the unique Kuhn-Tucker multiplier associated with it (i.e., $(x(\alpha), \lambda(\alpha))$ satisfies condition (2.2)).

The proof of Corollary 2.3 is given in the next section.

3. Proofs. We prepare the proofs of Theorem 2.1 by several lemmas. Some of them are more or less known. However the entire proofs are given for the sake of completeness. Our first lemma says that it is sufficient to prove Theorem 2.1 with Assumption (A.3) replaced by the stronger one

Assumption A.3. bis. There exist real numbers $a \geqq 0, c>0$ such that for all $h \in \mathbb{R}^{n}$ one has

$$
\left\langle D^{2} f\left(x^{0}, \alpha^{0}\right) h+\sum_{i=1}^{m} \lambda_{i}^{0} D^{2} g_{i}\left(x^{0}, \alpha^{0}\right) h, h\right\rangle+a \sum_{i=1}^{m_{1}}\left\langle\nabla g_{i}\left(x^{0}, \alpha^{0}\right), h\right\rangle^{2} \geqq c\|h\|^{2},
$$

(i.e. in (A.3.bis) the term $a\left\langle\nabla f\left(x^{0}, \alpha^{0}\right), h\right\rangle^{2}$ which appears in (A.3) has been removed.

LEMMA 3.0. If Theorem 2.1 is true when (A.3) is replaced by the stronger Assumption (A.3bis), then it is also true under Assumption (A.3).

Proof. Let us assume that the weak form of Theorem 2.1 holds (i.e. with Assumption (A.3) replaced by (A.3bis)). We show that Theorem 2.1 also holds. Let $\left(x^{0}, \alpha^{0}, \lambda^{0}\right) \in$ $U \times P \times \mathbb{R}^{m}$ satisfy Assumptions (A.0), (A.1), (A.2), (A.3), (A.4) together with the necessary conditions $(2.1)$ associated with the problem:

$$
\begin{gathered}
\text { minimize } f(x, \alpha) \\
\text { subject to } g(x, \alpha) \in Q, \\
x \in U .
\end{gathered}
$$

We now associate to all $\alpha \in P$ the following modified problem:

minimize $v$

$$
\begin{aligned}
\tilde{P}(\alpha) \quad \text { subject to } & f(x, \alpha)-v=0, \\
& g(x, \alpha) \in Q, \\
& (v, x) \in \mathbb{R} \times U .
\end{aligned}
$$

Clearly $(v, x)$ is a solution of $\tilde{\mathscr{P}}(\alpha)$ if and only if $x$ is a solution of $\mathscr{P}(\alpha)$ and $v=f(x, \alpha)$. Moreover at this solution $[(v, x),(\mu, \lambda)]$ satisfies the necessary conditions (2.1) associated with $\tilde{\mathscr{P}}(\alpha)$ if and only if $\mu=1,(x, \lambda)$ satisfies the necessary conditions (2.1) associated with $P(\alpha)$ and $v=f(x, \alpha)$.

Hence $\left(\left(v^{0}, x^{0}\right),\left(1, \lambda^{0}\right)\right)$, with $v^{0}=f\left(x^{0}, \alpha^{0}\right)$ satisfies the necessary conditions (2.1) associated with $\tilde{P}\left(\alpha^{0}\right)$. From the fact that $\left(x^{0}, \alpha^{0}, \lambda^{0}\right)$ satisfies Assumptions (A.0), (A.1), (A.2), (A.3) and (A.4) for problem $\tilde{\mathscr{P}}\left(\alpha^{0}\right)$ one deduces that $\left(\left(v^{0}, x^{0}\right),\left(1, \lambda^{0}\right)\right)$ satisfies Assumptions (A.0), (A.1), (A.2), (A.3bis) and (A.4) for problem $\tilde{\mathscr{P}}\left(\alpha^{0}\right)$. Applying the weak form of Theorem 2.1 to $\left(\left(v^{0}, x^{0}\right),\left(1, \lambda^{0}\right)\right)$ and using the above equivalence property between $\mathscr{P}(\alpha)$ and $\tilde{\mathscr{P}}(\alpha)$ one deduces the end of the proof of Lemma 3.0.

In the sequel we shall assume that $\left(x^{0}, \alpha^{0}, \lambda^{0}\right)$ satisfies (A.0), (A.1), (A.2), (A.3bis) and (A.4). 
LeMma 3.1. There exist neighborhoods $U_{1}$ of $x^{0}, U_{1} \subset U, V_{1}$ of $\alpha^{0}$ such that, for all $\alpha \in V_{1}$, if $x \in U_{1}$ is a local minimizer of $P(\alpha)$, then there exists $\lambda=\left(\lambda_{i}\right) \in \mathbb{R}^{m}$ such that $(x, \lambda)$ satisfies the first order necessary conditions at $\alpha$, i.e.,

$$
\begin{aligned}
& \nabla f(x, \alpha)+\sum_{i=1}^{m} \lambda_{i} \nabla g_{i}(x, \alpha)=0, \\
& g(x, \alpha) \in Q \text { and } \lambda \in N_{Q}(g(x, \alpha)) .
\end{aligned}
$$

Proof. Since by (A.1) the gradients $\nabla g_{i}\left(x^{0}, \alpha^{0}\right), i \in\{1, \cdots, m\}$ are independent from (A.0), there exist neighborhoods $U_{1}$ of $x^{0}, U_{1} \subset U, V_{1}$ of $\alpha^{0}$ such that, for all $(x, \alpha) \in U_{1} \times V_{1}$, the vectors $\nabla g_{i}(x, \alpha), i \in\{1, \cdots, m\}$, are independent. In the sequel of this proof, since $\alpha$ is fixed, there is no ambiguity denoting $f(x, \alpha), \nabla f(x, \alpha), \cdots$, simply by $f(x), \nabla f(x), \cdots$.

Let $X=\left\{x \in U_{1} \mid g(x) \in Q\right\}$. If $x$ is a local minimizer of $P(\alpha)$, from Clarke (1975), for all $v \in T_{x}(x)$ one has $\langle-\nabla f(x), v) \leqq 0$. Hence from Rockafellar (1970, Cor. 16.3.2), it is sufficient to prove that the following inclusion holds:

$$
\left\{u \in \mathbb{R}^{n} \mid D g(x) u \in T_{Q}(g(x))\right\} \subset T_{X}(x) .
$$

Indeed, let $u \in \mathbb{R}^{n}$ be such that $D g(x) u \in T_{Q}(g(x))$ and let $\left\{\theta^{q}\right\} \subset(0, \infty),\left\{x^{q}\right\} \subset X$ be sequences such that $\theta^{q} \rightarrow 0$ and $x^{q} \rightarrow x$. From the definition of the tangent cone it is sufficient to show that there exists a sequence $\left\{u^{q}\right\} \subset \mathbb{R}^{n}$, such that $u^{q} \rightarrow u$ and, for all $q, x^{q}+\theta^{q} u^{q} \in X$. Let $v=D g(x) u$, then $v \in T_{Q}(g(x))$. Since, for all $q, g\left(x^{q}\right) \in Q$, and $g\left(x^{q}\right) \rightarrow g(x)$, there exists a sequence $\left\{v^{q}\right\} \subset \mathbb{R}^{m}$ such that, for all $q, g\left(x^{q}\right)+\theta^{q} v^{q} \in Q$ and $v^{q} \rightarrow v$. We can choose vectors $b_{m+1}, \cdots, b_{n}$ in $\mathbb{R}^{n}$ so that the vectors $\nabla g_{1}(x), \cdots, \nabla g_{m}(x), b_{m+1}, \cdots, b_{n}$, form a basis in $\mathbb{R}^{n}$, and we define the mapping $G: U_{1} \rightarrow \mathbb{R}^{n}$ by $G(y)=\left(g_{1}(y), \cdots, g_{m}(y),\left\langle b_{m+1}, y\right\rangle, \cdots,\left\langle b_{n}, y\right\rangle\right)$. Clearly, $G$ is continuously differentiable, and the derivative $D G(x)$ is nonsingular. Hence, by the inverse mapping theorem, there exists $q_{0}$ such that, for $q \geqq q_{0}$, there exists $\hat{x}^{q} \in U_{1}$ satisfying:

$$
\begin{gathered}
g\left(\hat{x}^{q}\right)=g\left(x^{q}\right)+\theta^{q} v^{q}, \\
\left\langle b_{i}, \hat{x}^{q}\right\rangle=\left\langle b_{i}, x^{q}\right\rangle+\theta^{q}\left\langle b_{i}, u\right\rangle \quad(i=m+1, \cdots, n),
\end{gathered}
$$

and such that $\hat{x}^{q} \rightarrow x$. For $q \geqq q_{0}$, let $u^{q}=\left(\hat{x}^{q}-x^{q}\right) / \theta^{q}$. Recall that, for all $q, g\left(x^{q}\right)+$ $\theta^{q} v^{q} \in Q$, hence, for $q \geqq q_{0}, g\left(\hat{x}^{q}\right)=g\left(x^{q}\right)+\theta^{q} v^{q} \in Q$; thus $\left\{\hat{x}^{q}\right\} \subset X$. Consequently, for $q \geqq q_{0}, x^{q}+\theta^{q} u^{q}=\hat{x}^{q} \in X$. To end the proof of the lemma, it suffices to show that $u^{q} \rightarrow u$. Indeed, from Taylor's theorem, for $q \geqq q_{0}$, one has $g\left(\hat{x}^{q}\right)-g\left(x^{q}\right)=$ $\left[\int_{Q}^{1} D g\left(x^{q}+t\left(\hat{x}^{q}-x^{q}\right)\right) d t\right]\left(\hat{x}^{q}-x^{q}\right)$. Dividing by $\theta^{q}>0$, one gets $v^{q}=$ $\left[\int_{0}^{1} D g\left(x^{q}+t\left(\hat{x}^{q}-x^{q}\right)\right) d t\right] u^{q}$ and one easily deduces that $\lim _{q \rightarrow \infty} D g(x) u^{q}=\lim _{q \rightarrow \infty} v^{q}$. Recall that $\lim _{q \rightarrow \infty} v^{q}=v=D g(x) u$; hence, for all $i \leqq m, \lim _{q \rightarrow \infty}\left\langle\nabla g_{i}(x), u^{q}\right\rangle=$ $\left\langle\nabla g_{i}(x), u\right\rangle$. Furthermore, for all $i \geqq m+1,\left\langle b_{i}, u^{q}\right\rangle=\left\langle b_{i},\left(\hat{x}^{q}-x^{q}\right) / \theta^{q}\right\rangle=\left\langle b_{i}, u\right\rangle$. Since the vectors $\left\{\nabla g_{1}(x), \cdots, \nabla g_{m}(x), b_{m+1}, \cdots, b_{n}\right\}$ are independent, one deduces that $u^{q} \rightarrow u$. This ends the proof of the lemma.

LeMmA 3.2. For all $\varepsilon>0$ and all $c^{\prime} \in(0, c)$, there exist positive real numbers $k_{1}, k_{2}$, a positive real number $\delta$ (independent of $\varepsilon$ ) and neighborhoods $U_{2}$ of $x^{0}, U_{2} \subset U, V_{2}$ of $\alpha^{0}$, such that the two following properties are satisfied.

(a) For all $(x, \alpha),(y, \beta),\left(x^{1}, \alpha^{1}\right),\left(x^{2}, \alpha^{2}\right)$ in $U_{2} \times V_{2}$ such that $g\left(x^{1}, \alpha^{1}\right) \in Q$, $g\left(x^{2}, \alpha^{2}\right) \in Q$, for all $\lambda \in B\left(\lambda^{0}, \delta\right)$ one has:

$$
\begin{aligned}
& \left\langle\left[D^{2} f(x, \alpha)+\sum_{i=1}^{m} \lambda_{i} D^{2} g_{i}(y, \beta)\right]\left(x^{2}-x^{1}\right),\left(x^{2}-x^{1}\right)\right\rangle \\
& \quad \geqq c^{\prime}\left\|x^{2}-x^{1}\right\|^{2}-k_{1}\left\|x^{2}-x^{1}\right\| d\left(\alpha^{2}, \alpha^{1}\right)-k_{1} d\left(\alpha^{2}, \alpha^{1}\right)^{2} .
\end{aligned}
$$


(b) For all $\left(x^{1}, \alpha^{1}\right),\left(x^{2}, \alpha^{2}\right)$ in $U_{2} \times V_{2}$, such that $g\left(x^{2}, \alpha^{2}\right) \in Q, g\left(x^{1}, \alpha^{1}\right) \in Q$, for all $\lambda^{2} \in N_{Q}\left(g\left(x^{2}, \alpha^{2}\right)\right)$ one has:

$$
\begin{aligned}
& \left\langle\lambda^{2}, g\left(x^{2}, \alpha^{2}\right)-g\left(x^{1}, \alpha^{1}\right)\right\rangle \\
& \geqq\left\|\lambda_{I}^{2}\right\| \cdot\left[-\frac{\rho}{2}\left[\left\|D g_{I}\left(x^{0}, \alpha^{0}\right)\right\|+\varepsilon\right]^{2} \cdot\left\|x^{2}-x^{1}\right\|^{2}-k_{2}\left\|x^{2}-x^{1}\right\| d\left(\alpha^{2}, \alpha^{1}\right)-k_{2} d\left(\alpha^{2}, \alpha^{1}\right)^{2}\right] .
\end{aligned}
$$

Proof. We first claim that, for all $\varepsilon>0$, there exist a positive real number $k^{\prime}$ and neighborhoods $U^{\prime}$ of $x^{0}, U^{\prime} \subset U, V^{\prime}$ of $\alpha^{0}$ such that, for all $\left(x^{1}, \alpha^{1}\right),\left(x^{2}, \alpha^{2}\right)$ in $U^{\prime} \times V^{\prime}$, for all $i \in\{1, \cdots, m\}$ one has:

$$
\left|g_{i}\left(x^{2}, \alpha^{2}\right)-g_{i}\left(x^{1}, \alpha^{1}\right)-\left\langle\nabla g_{i}\left(x^{0}, \alpha^{0}\right),\left(x^{2}-x^{1}\right)\right\rangle\right| \leqq \varepsilon\left\|x^{2}-x^{1}\right\|+k^{\prime} d\left(\alpha^{2}, \alpha^{1}\right)
$$

Indeed, for all $i \in\{1, \cdots, m\}$, from (A.0), for all $\varepsilon>0$, there exist open neighborhoods $U^{\prime}$ of $x^{0}, U^{\prime} \subset U, V^{\prime}$ of $\alpha^{0}$, such that $U^{\prime}$ is convex, and, for all $(x, \alpha) \in U^{\prime} \times V^{\prime}$, $\left\|\nabla g_{i}(x, \alpha)-\nabla g_{i}\left(x^{0}, \alpha^{0}\right)\right\|<\varepsilon$. Furthermore, without any loss of generality, we can assume that there exists a positive real number $k^{\prime}$ such that $g_{i}$ is Lipschitzian of constant $k^{\prime}$ on $U^{\prime} \times V^{\prime}$. Hence, from Taylor's theorem,

$$
\begin{aligned}
& \left|g_{i}\left(x^{2}, \alpha^{2}\right)-g_{i}\left(x^{1}, \alpha^{1}\right)-\nabla g_{i}\left(x^{0}, \alpha^{0}\right)\left(x^{2}-x^{1}\right)\right| \\
& \leqq \\
& \quad\left|g_{i}\left(x^{2}, \alpha^{2}\right)-g_{i}\left(x^{1}, \alpha^{2}\right)-\left\langle\left[\int_{0}^{1} \nabla g_{i}\left(x^{1}+t\left(x^{2}-x^{1}\right), \alpha^{2}\right) d t\right], x^{2}-x^{1}\right\rangle\right| \\
& \quad+\left|g_{i}\left(x^{1}, \alpha^{2}\right)-g_{i}\left(x^{1}, \alpha^{1}\right)\right| \\
& \quad+\int_{0}^{1}\left\|\nabla g_{i}\left(x^{1}+t\left(x^{2}-x^{1}\right), \alpha^{2}\right)-\nabla g_{i}\left(x^{0}, \alpha^{0}\right)\right\| d t \cdot\left\|x^{2}-x^{1}\right\| \\
& \leqq 0+k^{\prime} d\left(\alpha^{2}, \alpha^{1}\right)+\varepsilon\left\|x^{2}-x^{1}\right\| .
\end{aligned}
$$

(a) For all $c^{\prime} \in(0, c)$, let $c^{\prime \prime} \in\left(c^{\prime}, c\right)$. From (A.3.bis) and the continuity of the mappings $D^{2} f(\cdot, \cdot)$ and $D^{2} g_{i}(\cdot, \cdot)(i=1, \cdots, m)$, there exist neighborhoods $U^{\prime \prime}$ of $x^{0}, U^{\prime \prime} \subset U, V^{\prime \prime}$ of $\alpha^{0}$ and a positive real number $\delta$ such that, for all $(x, \alpha),(y, \beta)$ in $U^{\prime \prime} \times V^{\prime \prime}$, for all $\lambda \in B\left(\lambda^{0}, \delta\right)$ and all $h \in \mathbb{R}^{n}$, one has:

$$
\left\langle\left[D^{2} f(x, \alpha)+\sum_{i=1}^{m} \lambda_{i} D^{2} g_{i}(y, \beta)\right] h, h\right\rangle \geqq c^{\prime \prime}\|h\|^{2}-a \sum_{i=1}^{m_{1}}\left\langle\nabla g_{i}\left(x^{0}, \alpha^{0}\right), h\right\rangle^{2} .
$$

Let $\varepsilon>0$ be such that $c^{\prime \prime}-a \cdot m_{1} \cdot \varepsilon^{2} \geqq c^{\prime}$, and let $U^{\prime}, V^{\prime}$ be the neighborhoods associated with $\varepsilon$ in the above claim (3.1). Let $U_{2}=U^{\prime} \cap U^{\prime \prime}$ and $V_{2}=V^{\prime} \cap V^{\prime \prime}$. Recall that, for all $(x, \alpha) \in U \times V$ such that $g(x, \alpha) \in Q$, from (A.0.vi), for $i \in\left\{1, \cdots, m_{1}\right\}$, $g_{i}(x, \alpha)=0$. Hence the end of the proof follows easily from (3.1) and the above inequality.

(b) From the weak convexity Assumption (A.2) and the continuity of the functions $g_{i}(i=1, \cdots, m)$, there exist open neighborhoods $U^{\prime \prime}$ of $x^{0}, U^{\prime \prime} \subset U$, and $V^{\prime \prime}$ of $\alpha^{0}$ such that, for all $\left(x^{1}, \alpha^{1}\right),\left(x^{2}, \alpha^{2}\right)$ in $U^{\prime \prime} \times V^{\prime \prime}$ such that $g\left(x^{k}, \alpha^{k}\right) \in Q(k=1,2)$, and for all $\mu^{2} \in N_{Q}\left(g\left(x^{2}, \alpha^{2}\right)\right) \cap \bar{B}(0,1)$, one has:

$$
\left\langle\mu^{2}, g\left(x^{2}, \alpha^{2}\right)-g\left(x^{1}, \alpha^{1}\right)\right\rangle \geqq-\frac{\rho}{2}\left\|g\left(x^{2}, \alpha^{2}\right)-g\left(x^{1}, \alpha^{1}\right)\right\|^{2} .
$$

Take any $\varepsilon>0$ and let $U^{\prime}, V^{\prime}$ be the neighborhoods associated with $\varepsilon$ in the above claim (3.1). Let $U_{2}=U^{\prime} \cap U^{\prime \prime}$ and $V=V^{\prime} \cap V^{\prime \prime}$. From (3.1) and the above inequality, there exists $k_{2}$ such that

$$
\begin{aligned}
& \left\langle\mu^{2}, g\left(x^{2}, \alpha^{2}\right)-g\left(x^{1}, \alpha^{1}\right)\right\rangle \\
& \quad \geqq-\frac{\rho}{2}\left[\left\|D g_{I}\left(x^{0}, \alpha^{0}\right)\right\|+\varepsilon\right]^{2} \cdot\left\|x^{2}-x^{1}\right\|^{2}-k_{2}\left\|x^{2}-x^{1}\right\| \cdot d\left(\alpha^{2}, \alpha^{1}\right)-k_{2} d\left(\alpha^{2}, \alpha^{1}\right)^{2} .
\end{aligned}
$$


Now letting $\lambda^{2} \in N_{Q}\left(g\left(x^{2}, \alpha^{2}\right)\right)$, we let $\mu^{2}=\left(0, \lambda_{I}^{2} /\left\|\lambda_{I}^{2}\right\|\right)$ if $\lambda_{I}^{2} \neq 0$ and $\mu^{2}=0$ if $\lambda_{I}^{2}=0$. Since $Q=\{0\} \times C, \quad N_{Q}\left(g\left(x^{2}, \alpha^{2}\right)\right)=\mathbb{R}^{m_{1}} \times N_{C}\left(g_{I}\left(x^{2}, \alpha^{2}\right)\right)$ and since $N_{C}\left(g_{I}\left(x^{2}, \alpha^{2}\right)\right)$ is a cone, one deduces that $\mu^{2} \in N_{Q}\left(g\left(x^{2}, \alpha^{2}\right)\right) \cap \bar{B}(0,1)$. Applying the above inequality to $\mu^{2}$ and noticing that

$$
\left\langle\mu^{2}, g\left(x^{2}, \alpha^{2}\right)-g\left(x^{1}, \alpha^{1}\right)\right\rangle=\left(1 /\left\|\lambda_{I}^{2}\right\|\right)\left\langle\lambda^{2}, g\left(x^{2}, \alpha^{2}\right)-g\left(x^{1}, \alpha^{1}\right)\right\rangle,
$$

yields the inequality of Lemma 3.2(b).

LEMMA 3.3. Let us suppose that $\left(x^{0}, \lambda^{0}\right)$ satisfies the first order necessary condition at $\alpha^{0}$. Then there exist positive real numbers $r$ and $b$ such that $\bar{B}\left(x^{0}, r\right) \subset U$ and, for all $x \in \bar{B}\left(x^{0}, r\right)$ satisfying $g\left(x, \alpha^{0}\right) \in Q$, one has $f\left(x, \alpha^{0}\right) \geqq f\left(x^{0}, \alpha^{0}\right)+b\left\|x-x^{0}\right\|^{2}$.

Proof. For every $\lambda=\left(\lambda_{i}\right) \in \mathbb{R}^{m}$, let $L(\cdot, \lambda): U \rightarrow \mathbb{R}$ be the function defined by $L(x, \lambda)=f\left(x, \alpha^{0}\right)+\sum_{i=1}^{m} \lambda_{i} g_{i}\left(x, \alpha^{0}\right)$. From Taylor's theorem, one has:

$$
\begin{aligned}
L\left(x, \lambda^{0}\right)= & L\left(x^{0}, \lambda^{0}\right)+\left\langle\nabla L\left(x^{0}, \lambda^{0}\right), x-x^{0}\right\rangle \\
& +\int_{0}^{1}(1-t)\left\langle D^{2} L\left(x^{0}+t\left(x-x^{0}\right), \lambda^{0}\right)\left(x-x^{0}\right),\left(x-x^{0}\right)\right\rangle d t .
\end{aligned}
$$

Let $\rho>0$ be the constant of weak convexity defined by (A.2). From (A.4), $c>\rho\left\|\lambda_{I}^{0}\right\| \cdot\left\|D g_{I}\left(x^{0}, \alpha^{0}\right)\right\|^{2}$. Hence, there exist $c^{\prime} \in(0, c)$ and $\varepsilon>0$ such that, if $b=$ $c^{\prime} / 2-\rho / 2\left\|\lambda_{I}^{0}\right\|\left[\left\|D g_{I}\left(x^{0}, \alpha^{0}\right)\right\|+\varepsilon\right]^{2}$, then $b>0$. Let $U_{2}, V_{2}$ be the neighborhoods of $x^{0}$ and $\alpha^{0}$ associated with $c^{\prime}$ and $\varepsilon$ in Lemma 3.2, and let $r$ be a positive real number such that $\bar{B}\left(x^{0}, r\right) \subset U_{2}$. Since $\left(x^{0}, \alpha^{0}\right)$ satisfies the first order necessary condition (2.1) at $\alpha^{0}$, one deduces that $\nabla L\left(x^{0}, \lambda^{0}\right)=0$. From Lemma 3.2(a) (taking $\alpha^{1}=\alpha^{2}=\alpha^{0}$ ), for all $x \in \bar{B}\left(x^{0}, r\right)$ one deduces that:

$$
L\left(x, \lambda^{0}\right)-L\left(x^{0}, \lambda^{0}\right) \geqq \int_{0}^{1}(1-t) c^{\prime}\left\|x-x^{0}\right\|^{2} d t \geqq \frac{c^{\prime}}{2}\left\|x-x^{0}\right\|^{2},
$$

and, from the definition of $L$, one gets:

$$
f\left(x, \alpha^{0}\right)-f\left(x^{0}, \alpha^{0}\right) \geqq\left\langle\lambda^{0}, g\left(x^{0}, \alpha^{0}\right)-g\left(x, \alpha^{0}\right)\right\rangle+\left(c^{\prime} / 2\right)\left\|x-x^{0}\right\|^{2} .
$$

By Lemma 3.2(b) (taking $\alpha^{1}=\alpha^{2}=\alpha^{0}$ ), for all $x \in \bar{B}\left(x^{0}, r\right) \subset U_{2}$, one gets:

$$
\left\langle\lambda^{0}, g\left(x^{0}, \alpha^{0}\right)-g\left(x, \alpha^{0}\right)\right\rangle \geqq-(\rho / 2)\left\|\lambda_{I}^{0}\right\|\left[\left\|D g_{I}\left(x^{0}, \alpha^{0}\right)\right\|+\varepsilon\right]^{2} .
$$

Hence, from the two above inequalities and the definition of $b$, for all $x \in \bar{B}\left(x^{0}, r\right)$, one has $f\left(x, \alpha^{0}\right)-f\left(x^{0}, \alpha^{0}\right) \geqq b\left\|x-x^{0}\right\|^{2}$. This completes the proof.

LEMMA 3.4. Let $\left(x^{0}, \lambda^{0}\right) \in U \times \mathbb{R}^{m}$ satisfy the first order necessary condition (2.1) at $\alpha^{0}$. Then, for every neighborhood $U^{\prime}$ of $x^{0}, U^{\prime} \subset U$, there exist $r^{\prime}>0$ and a neighborhood $V^{\prime}$ of $\alpha^{0}$ such that $B\left(x^{0}, r^{\prime}\right) \subset U^{\prime}$ and, for all $\alpha \in V^{\prime}$, there exists a minimizer $x(\alpha)$ of $P(\alpha)$ in $B\left(x^{0}, r^{\prime}\right)$ (i.e., $x(\alpha) \in B\left(x^{0}, r^{\prime}\right), g(x(\alpha), \alpha) \in Q$ and for all $x \in B\left(r^{0}, r\right), g(x, \alpha) \in$ $Q$ one has $f(x(\alpha), \alpha) \leqq f(x, \alpha))$.

The above lemma is related to a previous result of Robinson (1982), proved when $Q$ is a closed convex cone.

Proof. Let $U^{\prime}$ be a neighborhood of $x^{0}, U^{\prime} \subset U$, and let $b, r$ be the positive real numbers defined by Lemma 3.3. There exists a positive real number $r^{\prime}<r$ and a neighborhood $V_{1}^{\prime}$ of $\alpha^{0}$ such that $\bar{B}\left(x^{0}, r^{\prime}\right) \subset U^{\prime}$ and $f$ is $k$-Lipschitzian on $\bar{B}\left(x^{0}, r^{\prime}\right) \times V_{1}^{\prime}$.

For all $\alpha \in P$, let $\Gamma(\alpha)=\left\{x \in \bar{B}\left(x^{0}, r^{\prime}\right) \mid g(x, \alpha) \in Q\right\}$. We claim that there exists a neighborhood $V_{2}^{\prime}$ of $\alpha^{0}$ such that, for all $\alpha$ in $V_{2}^{\prime}$, there exists $x(\alpha) \in \Gamma(\alpha)$ satisfying:

$$
f(x(\alpha), \alpha) \leqq f(x, \alpha) \quad \text { for all } x \in \Gamma(\alpha) .
$$


Since $f$ is continuous and, for all $\alpha$ in $P, \Gamma(\alpha)$ is a compact subset of $\mathbb{R}^{n}$, it is sufficient to prove that, for $\alpha$ in a neighborhood of $\alpha^{0}, \Gamma(\alpha)$ is nonempty. Indeed, by (A.1) the vectors $\nabla g_{i}\left(x^{0}, \alpha^{0}\right), i \in\{1, \cdots, m\}$, are independent. Hence, by the implicit function theorem (Schwartz (1967)), there exists a neighborhood $V_{2}^{\prime}$ of $\alpha^{0}$ and a continuous mapping $\varphi: V_{2}^{\prime} \rightarrow \bar{B}\left(x^{0}, r^{\prime}\right)$ such that $\varphi\left(\alpha^{0}\right)=x^{0}$ and, for all $\alpha \in V_{2}^{\prime}$, one has $g(\varphi(\alpha), \alpha)=$ $g\left(x^{0}, \alpha^{0}\right) \in Q$. This ends the proof of the claim.

We now claim that there exists a neighborhood $V^{\prime}$ of $\alpha^{0}, V^{\prime} \subset V_{2}^{\prime}$, such that, for all $\alpha \in V^{\prime}$, the element $x(\alpha)$ defined before satisfies $\left\|x(\alpha)-x^{0}\right\|<r^{\prime}$. Clearly, the proof of the claim will end the proof of the lemma. Recall that $f$ is $k$-Lipschitzian on $\bar{B}\left(x^{0}, r^{\prime}\right) \times V_{1}^{\prime}$. Let $V_{3}^{\prime}=V_{1}^{\prime} \cap B\left(\alpha^{0}, r^{\prime 2} b / 16 k\right)$ and let $\eta>0, \eta<\min \left\{r^{\prime} / 2, r^{\prime 2} b / 16 k\right\}$. Then, for all $x^{1}, x^{2} \in B\left(x^{0}, r^{\prime}\right)$ satisfying $\left\|x^{2}-x^{1}\right\|<\eta$, for all $\alpha \in V_{3}^{\prime}$, one has:

$$
f\left(x^{1}, \alpha\right)-f\left(x^{2}, \alpha^{0}\right) \leqq k\left[\eta+d\left(\alpha, \alpha^{0}\right)\right]<r^{\prime 2} b / 8 \text {. }
$$

Furthermore, the multivalued mapping $\alpha \rightarrow \Gamma(\alpha)=\left\{x \in \bar{B}\left(x^{0}, r^{\prime}\right) \mid g(x, \alpha) \in Q\right\}$, from $V_{2}^{\prime}$ to $\mathbb{R}^{n}$, is upper semicontinuous, with compact values. Hence, there exists a neighborhood $V_{4}^{\prime}$ of $\alpha^{0}, V_{4}^{\prime} \subset V_{2}^{\prime}$, such that, for all $\alpha \in V_{4}^{\prime}, \Gamma(\alpha) \subset B\left(\Gamma\left(\alpha^{0}\right), \eta\right)$ and (from the continuity of $\varphi) \varphi(\alpha) \in B\left(x^{0}, \eta\right)$.

Let $V^{\prime}=V_{1}^{\prime} \cap V_{2}^{\prime} \cap V_{3}^{\prime} \cap V_{4}^{\prime}$. We now show that $V^{\prime}$ satisfies the conclusion of the lemma. Recall that, for all $\alpha \in V^{\prime}, \varphi(\alpha) \in \Gamma(\alpha)$; thus, from the definition of $x(\alpha)$, one deduces that:

$$
f(\varphi(\alpha), \alpha) \geqq f(x(\alpha), \alpha) .
$$

On the other hand, for all $\alpha \in V^{\prime}, x(\alpha) \in \Gamma(\alpha) \subset B\left(\Gamma\left(\alpha^{0}\right), \eta\right)$. Hence, there exists $y^{0} \in \Gamma\left(\alpha^{0}\right)$ such that $\left\|y^{0}-x(\alpha)\right\|<\eta$. By Lemma 3.3, one has:

$$
f\left(y^{0}, \alpha^{0}\right) \geqq f\left(x^{0}, \alpha^{0}\right)+b\left\|y^{0}-x^{0}\right\|^{2} .
$$

Summing up the two above inequalities, for all $\alpha \in V^{\prime}$, one gets:

$$
b\left\|y^{0}-x^{0}\right\|^{2} \leqq f(\varphi(\alpha), \alpha)-f\left(x^{0}, \alpha^{0}\right)+f\left(y^{0}, \alpha^{0}\right)-f(x(\alpha), \alpha) .
$$

Let us recall that, for $\alpha \in V^{\prime},\left\|\varphi(\alpha)-x^{0}\right\|<\eta$ and $\left\|y^{0}-x(\alpha)\right\|<\eta$. Hence, from the above inequality and the inequality defining $\eta$, one gets $b\left\|y^{0}-x^{0}\right\|^{2} \leqq r^{\prime 2} b / 8+r^{\prime 2} b / 8$. Thus, $\left\|y^{0}-x^{0}\right\|<r^{\prime} / 2$, and $\left\|x(\alpha)-x^{0}\right\| \leqq\left\|x(\alpha)-y^{0}\right\|+\left\|y^{0}-x^{0}\right\| \leqq \eta+r^{\prime} / 2<r^{\prime}$. This ends the proof of the claim and the proof of the lemma.

In the following, we denote by $D g(x, \alpha)^{*}$ the $n \times m$ matrix whose columns are $\nabla g_{i}(x, \alpha)(i=1, \cdots, m)$, i.e., the transpose of the $m \times n$ matrix $D g(x, \alpha)$.

LEMMA 3.5. Let $\varepsilon$ be a positive real number. There exist a positive real number $k_{3}$ and neighborhoods $U_{3}$ of $x^{0}, U_{3} \subset U, V_{3}$ of $\alpha^{0}$ such that, for all $(x, \alpha) \in U_{3} \times V_{3}$, the matrix $\operatorname{Dg}(x, \alpha) \circ D g(x, \alpha)^{*}$ is nonsingular and the mapping $\varphi: U_{3} \times V_{3} \rightarrow \mathbb{R}^{m}$ defined by

$$
\varphi(x, \alpha)=\left[D g(x, \alpha) \circ D g(x, \alpha)^{*}\right]^{-1} \circ D g(x, \alpha) \nabla f(x, \alpha),
$$

satisfies the following properties:

$$
\begin{aligned}
& \left\|\varphi\left(x^{2}, \alpha^{2}\right)-\varphi\left(x^{1}, \alpha^{1}\right)\right\| \leqq k_{3}\left[\left\|x^{2}-x^{1}\right\|+d\left(\alpha^{2}, \alpha^{1}\right)\right] \quad \text { for all }\left(x^{1}, \alpha^{1}\right),\left(x^{2}, \alpha^{2}\right) \text { in } U_{3} \times V_{3} \\
& \left\|\varphi(x, \alpha)-\varphi\left(x^{0}, \alpha^{0}\right)\right\| \leqq \varepsilon, \quad \text { for all }(x, \alpha) \in U_{3} \times V_{3} .
\end{aligned}
$$

Proof. The proof is a straightforward consequence of the independence Assumption (A.1), using the fact that the mappings $\nabla f(\cdot, \cdot), \nabla g_{i}(\cdot, \cdot)(i=1, \cdots, m)$ are locally Lipschitzian and that the mappings $A \rightarrow\left(A \circ A^{*}\right)^{-1} \circ A$, defined on the set of $m \times n$ matrices of maximal rank, is infinitely differentiable, and hence locally Lipschitzian. 
Proof of Theorem 2.1. Let $c>0, \rho>0$ be the constants defined by (A.2) and (A.3). From (A.4), $c>\rho\left\|\lambda_{I}^{0}\right\| \cdot\left\|D g_{I}\left(x^{0}, \alpha^{0}\right)\right\|^{2}$. Hence, there exist $c^{\prime} \in(0, c)$ and $\varepsilon>0$ such that

$$
c^{\prime}>\rho\left[\left\|\lambda_{I}^{0}\right\|+\varepsilon\right]\left[\left\|D g_{I}\left(x^{0}, \alpha^{0}\right)\right\|+\varepsilon\right]^{2} \text {. }
$$

Let $\delta>0$ be the constant defined by Lemma 3.2; without any loss of generality, we can suppose that $\varepsilon<\delta$. From (A.0), there exist a positive real number $k$ and neighborhoods $U_{0}$ of $x^{0}, U_{0} \subset U, V_{0}$ of $\alpha^{0}$ such that all the mappings $g(\cdot, \cdot) D g(\cdot, \cdot)$ and $\nabla f(\cdot, \cdot)$ are $k$-Lipschitzian on $U_{0} \times V_{0}$. Furthermore, let $U_{1}, V_{1}$ (resp. $U_{2}, V_{2}$ and $U_{3}, V_{3}$ ) be the neighborhoods of $x^{0}$ and $\alpha^{0}$ associated, in Lemma 3.1 (resp. Lemma 3.2 and Lemma 3.5) with the constants $c^{\prime}$ and $\varepsilon$ as defined above.

Now, by Lemma 3.4, we associate with $U^{\prime}=U_{0} \cap U_{1} \cap U_{2} \cap U_{3}$ a positive real number $r^{\prime}$ and an open neighborhood $\tilde{V}$ of $\alpha^{0}$. We take $V^{\prime}=\tilde{V} \cap V_{0} \cap V_{1} \cap V_{2} \cap V_{3}$. Let $\alpha^{1}, \alpha^{2}$ be two elements in $V^{\prime}$. By Lemma 3.4, there exists a minimizer of $P\left(\alpha^{1}\right)$ (resp. $P\left(\alpha^{2}\right)$ ) in $B\left(x^{0}, r^{\prime}\right)$ not necessarily unique, that we denote by $x^{1}$ (resp. $x^{2}$ ). By Lemma 3.1, let $\lambda^{1} \in \mathbb{R}^{m}$ (resp. $\lambda^{2} \in \mathbb{R}^{m}$ ) be the Kuhn-Tucker multiplier associated with $x^{1}$ (resp. $\left.x^{2}\right)$, i.e., such that $\left(x^{h}, \lambda^{h}\right)(h=1,2)$ satisfies the first order necessary condition at $\alpha^{h}$ :

$$
\begin{aligned}
& -\nabla f\left(x^{h}, \alpha^{h}\right)=D g\left(x^{h}, \alpha^{h}\right)^{*} \lambda^{h}, \\
& g\left(x^{h}, \alpha^{h}\right) \in Q \text { and } \lambda^{h} \in N_{Q}\left(g\left(x^{h}, \alpha^{h}\right)\right) .
\end{aligned}
$$

To end the proof of the theorem, it suffices to show that $\left\|x^{2}-x^{1}\right\| \leqq K d\left(\alpha^{2}, \alpha^{1}\right)$ and $\left\|\lambda^{2}-\lambda^{1}\right\| \leqq K d\left(\alpha^{2}, \alpha^{1}\right)$, where $K$ is a positive real number independent of the choice of $\alpha^{1}, \alpha^{2}$ in $V^{\prime}$.

From the first part of the first order necessary condition and Lemma 3.5, for $h=1,2$, the matrix $D g\left(x^{h}, \alpha^{h}\right) \circ D g\left(x^{h}, \alpha^{h}\right)^{*}$ is invertible and one deduces that:

$$
\begin{gathered}
\lambda^{h}=-\left[D g\left(x^{h}, \alpha^{h}\right) \circ D g\left(x^{h}, \alpha^{h}\right)^{*}\right]^{-1} D g\left(x^{h}, \alpha^{h}\right) \nabla f\left(x^{h}, \alpha^{h}\right)\left(=\varphi\left(x^{h}, \alpha^{h}\right)\right), \\
\left\|\lambda^{2}-\lambda^{1}\right\| \leqq k_{3}\left[\left\|x^{2}-x^{1}\right\|+d\left(\alpha^{2}, \alpha^{1}\right)\right], \\
\left\|\lambda_{I}^{h}-\lambda_{I}^{0}\right\| \leqq \lambda^{h}-\lambda^{0} \| \leqq \varepsilon<\delta .
\end{gathered}
$$

From (3.4) and from Lemma 3.2(b), one deduces that:

$$
\begin{aligned}
& {\left[\left\|\lambda_{I}^{0}\right\|+\varepsilon\right]^{-1} \cdot\left\langle\lambda^{2}-\lambda^{1}, g\left(x^{2}, \alpha^{2}\right)-g\left(x^{1}, \alpha^{1}\right)\right\rangle} \\
& \quad \geqq-\rho\left[\left\|D g_{I}\left(x^{0}, \alpha^{0}\right)\right\|+\varepsilon\right]^{2}\left\|x^{2}-x^{1}\right\|^{2} \\
& \quad-2 k_{2}\left\|x^{2}-x^{1}\right\| d\left(\alpha^{2}, \alpha^{1}\right)-2 k_{2} d\left(\alpha^{2}, \alpha^{1}\right)^{2} .
\end{aligned}
$$

Furthermore,

$$
\left\langle\lambda^{2}-\lambda^{1}, g\left(x^{2}, \alpha^{2}\right)-g\left(x^{1}, \alpha^{1}\right)\right\rangle=A+B+C,
$$

where

$$
\begin{aligned}
& A=\left\langle\lambda^{2}-\lambda^{1}, g\left(x^{2}, \alpha^{2}\right)-g\left(x^{2}, \alpha^{1}\right)\right\rangle, \\
& B=\left\langle-\lambda^{2}, g\left(x^{1}, \alpha^{1}\right)-g\left(x^{2}, \alpha^{1}\right)-g\left(x^{1}, \alpha^{2}\right)+g\left(x^{2}, \alpha^{2}\right)\right\rangle, \\
& C=\left\langle-\lambda^{1}, g\left(x^{2}, \alpha^{1}\right)-g\left(x^{1}, \alpha^{1}\right)\right\rangle+\left\langle-\lambda^{2}, g\left(x^{1}, \alpha^{2}\right)-g\left(x^{2}, \alpha^{2}\right)\right\rangle,
\end{aligned}
$$

and we consider successively each one of the three terms. From (3.3), using the Cauchy-Schwarz inequality and the fact that $g$ is $k$-Lipschitzian on $U^{\prime} \times V^{\prime}$, one gets:

$$
A \leqq k k_{3}\left[\left\|x^{2}-x^{1}\right\|+d\left(\alpha^{2}, \alpha^{1}\right)\right] \cdot d\left(\alpha^{2}, \alpha^{1}\right) .
$$


By (3.4) the multiplier $\lambda^{2}$ is bounded by $\left\|\lambda^{0}\right\|+\varepsilon$; hence, from Taylor's theorem, using the Cauchy-Schwarz inequality, one gets:

$$
B \leqq\left[\left\|\lambda^{0}\right\|+\varepsilon\right]\left\|x^{2}-x^{1}\right\|\left\|\int_{0}^{1} D g\left(x^{2}+t\left(x^{1}-x^{2}\right), \alpha^{1}\right) d t-\int_{0}^{1} D g\left(x^{1}+u\left(x^{2}-x^{1}\right), \alpha^{2}\right) d u\right\| \text {. }
$$

Upon performing the change of variable $t=1-u$ in the second integral, and using the fact that the derivative $D g(\cdot, \cdot)$ is $k$-Lipschitzian on $U^{\prime} \times V^{\prime}$, one gets:

$$
\begin{aligned}
& B \leqq\left[\left\|\lambda^{0}\right\|+\varepsilon\right]\left\|x^{2}-x^{1}\right\| \cdot \int_{0}^{1}\left\|D g\left(x^{2}+t\left(x^{1}-x^{2}\right), \alpha^{1}\right)-D g\left(x^{2}+t\left(x^{1}-x^{2}\right), \alpha^{2}\right)\right\| d t, \\
& B \leqq k\left[\left\|\lambda^{0}\right\|+\varepsilon\right]\left\|x^{2}-x^{1}\right\| d\left(\alpha^{2}, \alpha^{1}\right) .
\end{aligned}
$$

From Taylor's theorem, one has:

$$
\begin{aligned}
C= & \left\langle-\lambda^{1}, D g\left(x^{1}, \alpha^{1}\right)\left(x^{2}-x^{1}\right)\right\rangle+\left\langle-\lambda^{2}, D g\left(x^{2}, \alpha^{2}\right)\left(x^{1}-x^{2}\right)\right\rangle \\
& -\left\langle\left[\int_{0}^{1}(1-t) \sum_{i=1}^{m} \lambda_{i}^{1} D^{2} g_{i}\left(x^{1}+t\left(x^{2}-x^{1}\right), \alpha^{1}\right) d t\right]\left(x^{2}-x^{1}\right),\left(x^{2}-x^{1}\right)\right\rangle \\
& -\left\langle\left[\int_{0}^{1}(1-t) \sum_{i=1}^{m} \lambda_{i}^{2} D^{2} g_{i}\left(x^{2}+t\left(x^{1}-x^{2}\right), \alpha^{2}\right) d t\right]\left(x^{2}-x^{1}\right),\left(x^{2}-x^{1}\right)\right\rangle .
\end{aligned}
$$

From the first order necessary condition, Taylor's theorem, and using the fact that $\nabla f(\cdot, \cdot)$ is $k$-Lipschitzian on $U^{\prime} \times V^{\prime}$, one gets:

$$
\begin{aligned}
\left\langle-\lambda^{1},\right. & \left.D g\left(x^{1}, \alpha^{1}\right)\left(x^{2}-x^{1}\right)\right\rangle+\left\langle-\lambda^{2}, D g\left(x^{2}, \alpha^{2}\right)\left(x^{1}-x^{2}\right)\right\rangle \\
= & -\left\langle\nabla f\left(x^{2}, \alpha^{2}\right)-\nabla f\left(x^{1}, \alpha^{1}\right), x^{2}-x^{1}\right\rangle \\
= & -\left\langle\nabla f\left(x^{2}, \alpha^{2}\right)-\nabla f\left(x^{2}, \alpha^{1}\right), x^{2}-x^{1}\right\rangle-\left\langle\nabla f\left(x^{2}, \alpha^{1}\right)-\nabla f\left(x^{1}, \alpha^{1}\right), x^{2}-x^{1}\right\rangle \\
\leqq & k d\left(\alpha^{2}, \alpha^{1}\right)\left\|x^{2}-x^{1}\right\| \\
& -\left\langle\left[\int_{0}^{1} D^{2} f\left(x^{1}+t\left(x^{2}-x^{1}\right), \alpha^{1}\right) d t\right]\left(x^{2}-x^{1}\right),\left(x^{2}-x^{1}\right)\right\rangle .
\end{aligned}
$$

One easily gets (by performing the change of variable $t=1-u$ ) that:

$$
\int_{0}^{1} t D^{2} f\left(x^{1}+t\left(x^{2}-x^{1}\right), \alpha^{1}\right) d t=\int_{0}^{1}(1-u) D^{2} f\left(x^{2}+u\left(x^{1}-x^{2}\right), \alpha^{1}\right) d u .
$$

Hence

$$
\begin{aligned}
\int_{0}^{1} D^{2} f\left(x^{1}+t\left(x^{2}-x^{1}\right), \alpha^{1}\right) d t= & \int_{0}^{1}(1-t) D^{2} f\left(x^{1}+t\left(x^{2}-x^{1}\right), \alpha^{1}\right) d t \\
& +\int_{0}^{1}(1-u) D^{2} f\left(x^{2}+u\left(x^{1}-x^{2}\right), \alpha^{1}\right) d u
\end{aligned}
$$

From (3.4), $\lambda^{h} \in \bar{B}\left(\lambda^{0}, \delta\right)(h=1,2)$. Hence, from Lemma 3.2(a) and the above equalities and inequalities

$$
\begin{aligned}
& C \leqq k d\left(\alpha^{2}, \alpha^{1}\right)\left\|x^{2}-x^{1}\right\| \\
& \quad-2 \int_{0}^{1}(1-t)\left[c^{\prime}\left\|x^{2}-x^{1}\right\|^{2}-k_{1}\left\|x^{2}-x^{1}\right\| d\left(\alpha^{2}, \alpha^{1}\right)-k_{1} d\left(\alpha^{2}, \alpha^{1}\right)^{2}\right] d t \\
& C \leqq-c^{\prime}\left\|x^{2}-x^{1}\right\|^{2}+\left(k+k_{1}\right)\left\|x^{2}-x^{1}\right\| d\left(\alpha^{2}, \alpha^{1}\right)+k_{1} d\left(\alpha^{2}, \alpha^{1}\right)^{2}
\end{aligned}
$$


Let $b=c^{\prime}-\rho\left[\left\|\lambda_{I}^{0}\right\|+\varepsilon\right]\left[\left\|D g_{I}\left(x^{0}, \alpha^{0}\right)\right\|+\varepsilon\right]^{2}$; then, from (3.2) one has $b>0$. From (3.5), (3.6), (3.7), (3.8), (3.9), there exists a positive real number $k^{\prime}$ such that:

$$
b\left\|x^{2}-x^{1}\right\|^{2} \leqq k^{\prime} d\left(\alpha^{2}, \alpha^{1}\right)\left[\left\|x^{2}-x^{1}\right\|+d\left(\alpha^{2}, \alpha^{1}\right)\right] .
$$

Let $K=\max \left\{2,\left(2 k^{\prime}\right) / b\right\}$. Then, from the above inequality, one easily deduces that $\left\|x^{2}-x^{1}\right\| \leqq K d\left(\alpha^{2}, \alpha^{1}\right)$, and, from (3.3), $\left\|\lambda^{2}-\lambda^{1}\right\| \leqq\left(k_{3}+K k_{3}\right) d\left(\alpha^{2}, \alpha^{1}\right)$. This ends the proof of the theorem.

We now give the proof of Corollary 2.3.

Proof of Corollary 2.3. Let $\left(x^{0}, \alpha^{0}, \lambda^{0}\right)$ satisfy the assumptions of Corollary 2.3 and let:

$$
\begin{aligned}
& I_{+}\left(x^{0}, \alpha^{0}\right)=\left\{i \in I\left(x^{0}, \alpha^{0}\right) \mid \lambda_{i}^{0}>0\right\}, \quad I_{0}\left(x^{0}, \alpha^{0}\right)=\left\{i \in I\left(x^{0}, \alpha^{0}\right) \mid \lambda_{i}^{0}=0\right\}, \\
& J\left(x^{0}, \alpha^{0}\right)=\left\{i \in\left\{m_{1}+1, \cdots, m\right\} \mid g_{i}\left(x^{0}, \alpha^{0}\right)<0\right\} .
\end{aligned}
$$

For $\alpha \in P$, we consider the following problem:

minimize $f(x, \alpha)$

$\tilde{S}(\alpha)$

$$
\text { subject to } g_{i}(x, \alpha)=0, i \in\left\{1, \cdots, m_{1}\right\} \cup I_{+}\left(x^{0}, \alpha^{0}\right) \text {, }
$$

$$
g_{i}(x, \alpha) \leqq 0, i \in I_{0}\left(x^{0}, \alpha^{0}\right),
$$

$x \in U$.

Clearly $\tilde{S}(\alpha)$ is a problem of type $P(\alpha)$ with $Q=\{0\}^{p} x\left(-\mathbb{R}_{+}\right)^{q}$, where $p=$ card $I_{+}\left(x^{0}, \alpha^{0}\right)+m_{1}$ and $q=$ card $I_{0}\left(x^{0}, \alpha^{0}\right)$. If we let $\tilde{\lambda}^{0}$ be the vector in $\mathbb{R}^{p+q}$ with coordinates $\tilde{\lambda}_{i}^{0}=\lambda_{i}^{0}$, for $i \in\left\{1, \cdots, m_{1}\right\} \cup I\left(x^{0}, \alpha^{0}\right)$, then $\left(x^{0}, \alpha^{0}, \tilde{\lambda}^{0}\right)$ satisfies the assumptions of Theorem 2.1. Furthermore, $\left(x^{0}, \alpha^{0}, \tilde{\lambda}^{0}\right)$ satisfies the necessary condition (2.2) associated with $\tilde{S}\left(\alpha^{0}\right)$, since $\left(x^{0}, \alpha^{0}, \lambda^{0}\right)$ satisfies the necessary condition (2.2) associated with $S\left(\alpha^{0}\right)$. Consequently, from Theorem 2.1, there exist open neighborhoods $U_{1}$ of $x^{0}$ in $U, V_{1}$ of $\alpha^{0}$ in $P$ and Lipschitzian mappings $x(\cdot): V_{1} \rightarrow U_{1}$ and $\tilde{\lambda}(\cdot): V_{1} \rightarrow \mathbb{R}^{p+q}$ such that $x\left(\alpha^{0}\right)=x^{0}, \tilde{\lambda}\left(\alpha^{0}\right)=\tilde{\lambda}^{0}$ and, for all $\alpha \in V_{1}$, the pair $(x(\alpha), \tilde{\lambda}(\alpha))$ satisfies the necessary condition (2.2) associated with problem $\tilde{S}(\alpha)$. We let now $\lambda(\cdot): V_{1} \rightarrow \mathbb{R}^{m}$ be the mapping defined by $\lambda_{i}(\alpha)=\tilde{\lambda}_{i}(\alpha)$ for $i \in\left\{1, \cdots, m_{1}\right\} \cup I\left(x^{0}, \alpha^{0}\right)$ and $\lambda_{i}(\alpha)=0$ for $i \in J\left(x^{0}, \alpha^{0}\right)$. Since, for all $i \in I_{+}\left(x^{0}, \alpha^{0}\right), \lambda_{i}\left(\alpha^{0}\right)=\lambda_{i}^{0}>0$ and, for all $i \in J\left(x^{0}, \alpha^{0}\right), g_{i}\left(x^{0}, \alpha^{0}\right)=g_{i}\left(x\left(\alpha^{0}\right), \alpha^{0}\right)<0$, from the continuity of the mappings $\lambda(\cdot), x(\cdot)$ and $g_{i}(\cdot, \cdot)$, there exists an open neighborhood $V_{2}$ of $\alpha^{0}$ in $V_{1}$ such that, for all $\alpha$ in $V_{2}$, for all $i \in I_{+}\left(x^{0}, \alpha^{0}\right), \lambda_{i}(\alpha)>0$ and, for all $i \in J\left(x^{0}, \alpha^{0}\right), g_{i}(x(\alpha), \alpha)<0$. Consequently, one easily checks that, for all $\alpha \in V_{2},(x(\alpha), \lambda(\alpha))$ satisfies the necessary condition (2.2) associated with problem $S(\alpha)$.

It now remains to show that there exist neighborhoods $V^{\prime}$ of $\alpha^{0}$ in $V_{2}$ and $U^{\prime}$ of $x^{0}$ in $U_{1}$ such that, for all $\alpha \in V^{\prime}$ and all $x \in U^{\prime}, x \neq x(\alpha)$, satisfying $g_{i}(x, \alpha)=0$ for $i \in\left\{1, \cdots, m_{1}\right\}$ and, $g_{i}(x, \alpha) \leqq 0$, for $i \in\left\{m_{1}+1, \cdots, m\right\}$, then one has $f(x, \alpha)>$ $f(x(\alpha), \alpha)$. We prove this assertion by contraposition. Assume that there exist sequences $\left\{x^{k}\right\} \subset U^{\prime}$ and $\left\{\alpha^{k}\right\} \subset V^{\prime}$ such that $\left\{x^{k}\right\} \rightarrow x^{0},\left\{\alpha^{k}\right\} \rightarrow \alpha^{0}$ and, for all $k, x^{k} \neq$ $x\left(\alpha^{k}\right), g_{i}\left(x^{k}, \alpha^{k}\right)=0$ for $i \in\left\{1, \cdots, m_{1}\right\}, g_{i}\left(x^{k}, \alpha^{k}\right) \leqq 0$ for $i \in\left\{m_{1}+1, \cdots, m\right\}$ and $f\left(x^{k}, \alpha^{k}\right) \leqq f\left(x\left(\alpha^{k}\right), \alpha^{k}\right)$. Without any loss of generality, one can assume that $\left(x^{k}-\right.$ $\left.x\left(\alpha^{k}\right)\right) /\left\|x^{k}-x\left(\alpha^{k}\right)\right\|$ converges to some element $h$ in $\mathbb{R}^{n}$ such that $\|h\|=1$.

From the mean value theorem and the continuity of $\nabla f(\cdot, \cdot)$ and $\nabla g_{i}(\cdot, \cdot)$ at $\left(x^{0}, \alpha^{0}\right)$ one has, for $i=1, \cdots, m$,

$$
\begin{aligned}
& \left\langle\nabla f\left(x^{0}, \alpha^{0}\right), h\right\rangle=\lim _{k \rightarrow \infty}\left[f\left(x^{k}, \alpha^{k}\right)-f\left(x\left(\alpha^{k}\right), \alpha^{k}\right)\right] /\left\|x^{k}-x\left(\alpha^{k}\right)\right\|, \\
& \left\langle\nabla g_{i}\left(x^{0}, \alpha^{0}\right), h\right\rangle=\lim _{k \rightarrow \infty}\left[g_{i}\left(x^{k}, \alpha^{k}\right)-g_{i}\left(x\left(\alpha^{k}\right), \alpha^{k}\right)\right] /\left\|x^{k}-x\left(\alpha^{k}\right)\right\| .
\end{aligned}
$$


From the very definition of the sequences $\left\{x^{k}\right\}$ and $\left\{\alpha^{k}\right\}$ and recalling that from the first part of the proof, $g_{i}\left(x\left(\alpha^{k}\right), \alpha^{k}\right)=0, i=\left\{1, \cdots, m_{1}\right\} \cup I_{+}\left(x^{0}, \alpha^{0}\right)$, one deduces from (3.10) that

$$
\begin{array}{ll}
\left\langle\nabla f\left(x^{0}, \alpha^{0}\right), h\right\rangle \leqq 0, & \\
\left\langle\nabla g_{i}\left(x^{0}, \alpha^{0}\right), h\right\rangle=0, & i=1, \cdots, m_{1}, \\
\left\langle\nabla g_{i}\left(x^{0}, \alpha^{0}\right), h\right\rangle \leqq 0, & i \in I_{+}\left(x^{0}, \alpha^{0}\right) .
\end{array}
$$

Since $\left(x^{0}, \lambda^{0}\right)$ satisfies the necessary condition (2.2) associated with $S\left(\alpha^{0}\right)$, one gets

$$
\left\langle\nabla f\left(x^{0}, \alpha^{0}\right), h\right\rangle+\sum_{i \in\left\{1, \cdots, m_{1}\right\} \cup I_{+}\left(x^{0}, \alpha^{0}\right)} \lambda_{i}^{0}\left\langle\nabla g_{i}\left(x^{0}, \alpha^{0}\right), h\right\rangle=0 .
$$

From (3.11) and (3.12), since $\lambda_{i}^{0}>0$, for $i \in I_{+}\left(x^{0}, \alpha^{0}\right)$, it follows that the inequalities in (3.11) are in fact equalities. Hence, by (C.2) one gets

$$
\left\langle D^{2} f\left(x^{0}, \alpha^{0}\right) h+\sum_{i=1}^{m} \lambda_{i}^{0} D^{2} g_{i}\left(x^{0}, \alpha^{0}\right) h, h\right\rangle>0 .
$$

We end the proof of the corollary by contradicting (3.13). Denote $L_{k}(x)=$ $f\left(x, \alpha^{k}\right)+\sum_{i=1}^{m} \lambda_{i}\left(\alpha^{k}\right) g_{i}\left(x, \alpha^{k}\right)$. From Taylor's theorem and from the continuity of $D^{2} f(\cdot, \cdot)$ and $D^{2} g_{i}(\cdot, \cdot)$ at $\left(x^{0}, \alpha^{0}\right)$ :

$$
\begin{aligned}
& \frac{1}{2}\left\langle D^{2} f\left(x^{0}, \alpha^{0}\right) h+\sum_{i=1}^{m} \lambda_{i}^{0} D^{2} g_{i}\left(x^{0}, \alpha^{0}\right) h, h\right\rangle \\
& \quad=\lim _{k \rightarrow \infty} \int_{0}^{1}(1-t)\left\langle D^{2} L_{k}\left[x\left(\alpha^{k}\right)+t\left(x^{k}-x\left(\alpha^{k}\right)\right)\right] \frac{\left(x^{k}-x\left(\alpha^{k}\right)\right)}{\left\|x^{k}-x\left(\alpha^{k}\right)\right\|}, \frac{x^{k}-x\left(\alpha^{k}\right)}{\left\|x^{k}-x\left(\alpha^{k}\right)\right\|}\right\rangle d t \\
& \quad=\lim _{k \rightarrow \infty}\left[L_{k}\left(x^{k}\right)-L_{k}\left(x\left(\alpha^{k}\right)\right)-\left\langle\nabla L_{k}\left(x\left(\alpha^{k}\right)\right), x^{k}-x\left(\alpha^{k}\right)\right\rangle\right] /\left\|x^{k}-x\left(\alpha^{k}\right)\right\|^{2},
\end{aligned}
$$

where

$$
h=\lim \frac{x^{k}-x\left(\alpha^{k}\right)}{\left\|x^{k}-x\left(\alpha^{k}\right)\right\|} .
$$

Since $\left(x\left(\alpha^{k}\right), \lambda\left(\alpha^{k}\right)\right)$ satisfies the necessary conditions (2.2) associated with $S\left(\alpha^{k}\right)$, then $\nabla L_{k}\left(x\left(\alpha^{k}\right)\right)=0, \lambda_{i}\left(\alpha^{k}\right) g_{i}\left(x\left(\alpha^{k}\right), \alpha^{k}\right)=0$, for all $i \in\{1, \cdots, m\}$ and $\lambda_{i}\left(\alpha^{k}\right) \geqq 0$, for $i \in\left\{m_{1}+1, \cdots, m\right\}$. Consequently, $L_{k}\left(x\left(\alpha^{k}\right)\right)=f\left(x\left(\alpha^{k}\right)\right)$ and $L_{k}\left(x^{k}\right)=$ $f\left(x^{k}\right)+\sum_{i=1}^{m} \lambda_{i}\left(\alpha^{k}\right) g_{i}\left(x^{k}, \alpha^{k}\right) \leqq f\left(x^{k}\right)$. Hence, for all $k$,

$$
L_{k}\left(x^{k}\right)-L_{k}\left(x\left(\alpha^{k}\right)\right)-\left\langle\nabla L_{k}\left(x\left(\alpha^{k}\right)\right), x^{k}-x\left(\alpha^{k}\right)\right\rangle \leqq 0 .
$$

Dividing the above inequality by $\left\|x^{k}-x\left(\alpha^{k}\right)\right\|^{2}$, passing to the limit, when $k \rightarrow \infty$, from the above equalities one deduces that:

$$
\left\langle D^{2} f\left(x^{0}, \alpha^{0}\right) h+\sum_{i=1}^{m} \lambda_{i}^{0} D^{2} g_{i}\left(x^{0}, \alpha^{0}\right) h, h\right\rangle \leqq 0
$$

which contradicts (3.13). This ends the proof of the corollary.

\section{REFERENCES}

[1] J. P. AUBIN, Lipschitz behaviour of solutions to convex minimization problems, Working Paper, IASA, 1981.

[2] A. AUSLENDER, Theorem of constant rank for Lipschitzian maps and applications in optimization theory, in Mathematical Programming with Data Perturbations, Vol. 2, A. V. Fiacco, ed., Marcel Dekker, New York, 1983. 
[3] F. Clarke, Generalized gradients and applications, Trans. Amer. Math. Soc., 205 (1975), pp. $247-262$.

[4] - On the inverse function theorem, Pacific J. Math., 64 (1976), pp. 97-102.

[5] B. CORNET AND G. LAROQUE, Lipschitz properties of constrained demand functions and constrained maximizers, INSEE Working Paper 8005, 1980.

[6] B. CORnet, Contributions à la théorie des mécanismes dynamiques d'allocation des ressources, thèse d'état, Université Paris IX Dauphine, 1981.

[7] B. CORNET AND G. LAROQUE, Lipschitz properties of solutions in mathematical programming, J. Optim. Theory Appl., 1986, to appear.

[8] G. Debreu, Definite and semi-definite quadratic forms, Econometrica, 20 (1952), pp. 295-299.

[9] A. V. FIACCO AND G. P. MCCormICK, Nonlinear Programming: Sequential Unconstrained Minimization Techniques, John Wiley, New York, 1968.

[10] A. V. FIACCO, Sensitivity analysis for nonlinear programming using penalty methods, Math. Programming, 10 (1976), pp. 287-311.

[11] A. V. FIACCO AND W. P. HUTZLER, Basic results in the development of sensitivity and stability analysis in nonlinear programming, Comput. Oper. Res., 9, 1 (1982), pp. 9-28.

[12] J. B. HIRIART-URRUTY, Tangent cones, generalized gradients and mathematical programming in Banach spaces, Math. Oper. Res., 4, 1 (1979), pp. 79-97.

[13] K. JITTORNTRUM, Solution point differentiability without strict complementarity in nonlinear programming, Math. Programming, 21 (1984), pp. 127-138.

[14] M. KoJima, Strongly stable stationary solutions in nonlinear programs, in Analysis and Computation of Fixed Points, S. M. Robinson, ed., Academic Press, New York, 1980, pp. 93-138.

[15] E. S. LEVITIN, On the local perturbation theory of a problem of mathematical programming in a Banach space, Soviet Math. Dokl., 15 (1975), pp. 603-608.

[16] S. M. RoBINSON, Perturbed Kuhn-Tucker points and rates of convergence for a class of nonlinear programming algorithms, Math. Programming, 7 (1974), pp. 1-16.

[17] - Strongly regular generalized equations, Math. Oper. Res., 5, 1 (1980), pp. 43-62.

[18] - Generalized equations and their solution, Part II: Applications to nonlinear programming, in Optimality and Stability in Mathematical Programming, M. Guignard, ed., Mathematical Programming Study, 19 (1982).

[19] R. T. Rockafellar, Convex Analysis, Princeton Univ. Press, Princeton, NJ, 1970.

[20] - The Theory of Subgradients and its Applications to Problems of Optimization, Convex and Nonconvex Functions, Helderman-Verlag, Berlin, 1981.

[21] L. Schwartz, Analyse mathématique, Cours de l'Ecole Polytechnique, Hermann, Paris, 1967.

[22] J. P. VIAL, Strong and weak convexity of sets and functions, Math. Oper. Res., 8 (1983), pp. 231-259. 